\title{
Realization of a timescale with an accurate optical lattice clock: supplementary material
}

\author{
Christian Grebing*, Ali Al-Masoudi, Sören Dörscher, Sebastian HäFner, \\ Vladislav Gerginov, Stefan Weyers, Burghard Lipphardt, Fritz Riehle, \\ UWE STERR, AND CHRISTIAN LISDAT
}

Physikalisch-Technische Bundesanstalt, Bundesallee 100, 38116 Braunschweig, Germany

*Corresponding author: christian.grebing@ptb.de

Published 25 May 2016

\begin{abstract}
This document contains supplementary information on the optical Sr clock operation, its uncertainty budget and the theory of data analysis for "Realization of a timescale with an accurate optical lattice clock," http://dx.doi.org/10.1364/optica.3.000563.
\end{abstract}

(C) 2016 Optical Society of America

http://dx.doi.org/10.1364/optica.3.000563.s001

Sr lattice clock operation To interrogate the $698 \mathrm{~nm}^{1} S_{0}-{ }^{3} P_{0}$ clock transition of ${ }^{87} \mathrm{Sr}$, an atomic beam from an oven is Zeemanslowed and loaded into a magneto-optical trap (MOT) operated on the strong $461 \mathrm{~nm}{ }^{1} S_{0}-{ }^{1} P_{1}$ transition. After less than $300 \mathrm{~ms}$ loading, the atoms are transferred into a second-stage MOT operated on the $689 \mathrm{~nm}$ intercombination line, ${ }^{1} S_{0}-{ }^{3} P_{1}$, that allows for laser cooling of the atoms to a few microkelvin.

During the last cooling phase, the atoms are loaded into a nearly horizontally oriented 1D-optical lattice at the Stark-shift cancellation wavelength of strontium near $813 \mathrm{~nm}$ [1]. In the lattice, the atoms are spin-polarized to either the $m_{f}=+9 / 2$ or $-9 / 2$ level of the ground state. The polarization of the sample is purified by a short $\pi$ pulse on the clock transition in a homogeneous magnetic bias field and subsequent removal of remaining ground state atoms.

After removing high-energy atoms from the lattice by temporarily reducing the lattice depth [2], the clock transition is interrogated in a bias field of about $25 \mu \mathrm{T}$. The excitation probability is estimated from the atoms in ground and excited state after the interrogation. To derive an error signal free from linear Zeeman shift the $m_{f}= \pm 9 / 2$ transitions are sequentially interrogated at both points of maximum slope; thus four interrogations update the error signal. A single interrogation sequence lasts about $1 \mathrm{~s}$, or, if a dead time is introduced to reduce heat load and thermal gradients, one interrogation every $2 \mathrm{~s}$ is performed.

Different from our previous set-up [2], the $429 \mathrm{THz}$ clock laser system interrogating the ${ }^{87} \mathrm{Sr}^{1} S_{0}-{ }^{3} P_{0}$ clock transition was replaced such that stabilization to the cryogenic silicon cavity [3] is no longer required [4]. The new clock laser system uses a
$48 \mathrm{~cm}$ long reference resonator made from ultra-low expansion glass [5]. It enables regular coherent interrogation of $640 \mathrm{~ms}$ duration, which leads to a resonance linewidth of about $1.4 \mathrm{~Hz}$ full-width at half-maximum.

Sr lattice clock uncertainty contributions

BBR shift: The Stark shift caused by BBR was determined experimentally [6], and validated theoretically [7] as well as experimentally [8]. Recently, the dynamic correction factor $v_{\text {dyn }}$, reflecting the difference in frequency shift between BBR and a static electric field with equal rms amplitude, was refined [9]. In our experiment, the uncertainty due to BBR is limited by the uncertainty of the representative temperature $T_{\text {rep }}$. Temperature differences of $1.3 \mathrm{~K}$ at maximum measured between the warmest $\left(T_{\max }\right)$ and coldest $\left(T_{\min }\right)$ point of the apparatus lead under the assumption of a uniform probability distribution of $T_{\text {rep }}$ to the most probable temperature of $\left(T_{\max }+T_{\min }\right) / 2 \approx 294 \mathrm{~K}$ and an uncertainty of $1.28 \times 10^{-17}$ of the fractional frequency correction of $492.9 \times 10^{-17}$. In addition, radiation from the hot oven reaches the atoms and we estimate a corresponding correction of $9.4(9.4) \times 10^{-18}$ [2].

Zeeman shifts: The linear Zeeman shift, as well as the vector light shift, are removed by stabilizing the interrogation laser to the mean frequency of the $m_{F}= \pm 9 / 2$ Zeeman components [10]. Moreover, this scheme provides continuous monitoring of the total magnetic field experienced by the atoms and thus enables a characterization and correction of the second-order Zeeman shift. We find a shift of $-36(1.5) \times 10^{-18}$. The uncertainty is governed by the uncertainty of the magnetic field derived from the line splitting. The uncertainty of the correction coefficient 


\begin{tabular}{lcl}
\hline effect & $\begin{array}{c}\text { correction } \\
\left(10^{-17}\right)\end{array}$ & $\begin{array}{c}\text { uncertainty } \\
\left(10^{-17}\right)\end{array}$ \\
\hline \hline BBR room & 492.9 & 1.28 \\
BBR oven & 0.94 & 0.94 \\
second-order Zeeman & 3.6 & 0.15 \\
cold collisions & 0 & 0.08 \\
background gas collisions & 0 & 0.4 \\
line pulling & 0 & 0.01 \\
lattice scalar/tensor & -0.7 & 0.9 \\
lattice E2/M1 & 0 & 0.34 \\
hyperpolarisability & -0.39 & 0.18 \\
tunnelling & 0 & 0.21 \\
probe light & 0 & 0.01 \\
optical path length error & 0 & 0.01 \\
servo error & 0 & 0.17 \\
DC Stark shift & 0 & 0.03 \\
\hline total & 496.4 & 1.9 \\
\hline
\end{tabular}

Table S1. Corrections applied to the measured Sr lattice clock frequency and their uncertainties during the June 2015 frequency measurement given in fractional units. Details are given in the text.

and the vector light shift can be neglected.

Collisions: Cold collisions and collisions with the background gas may cause frequency shifts. We measured the cold collision shift by variation of the atom density in the lattice and found a shift of $0(8) \times 10^{-19}$. From the lattice lifetime of $4 \mathrm{~s}$, background gas collisions lead to a shift of $0(4) \times 10^{-18}$ based on [11].

Line pulling: Line pulling is induced by overlapping transitions caused by atoms populating different levels or by polarization imperfections of the excitation laser such that not only $\pi$ but also $\sigma$-transitions are driven simultaneously. Investigation of the preparation sequence and the laser polarization as seen by the atoms allows us to assign a negligible correction with small uncertainty to the line pulling effect of $0(1) \times 10^{-19}$.

Lattice light shifts: By varying the lattice depth between the usual spectroscopy setting of $72 E_{r}$ and $110 E_{r}$, with the recoil energy of the lattice $E_{r}$, we find a Stark shift cancellation frequency of 368554 471(3) MHz for mutually parallel lattice polarization and magnetic field orientation. During the measurement campaign the lattice light frequency is typically slightly offset against the Stark cancellation frequency, which leads to a shift of the clock frequency of $7(9) \times 10^{-18}$. Besides the determination of the Stark shift cancellation frequency under the given experimental conditions of lattice polarization and orientation, possible shifts due to multi-photon and higher-order multi-pole excitations have to be accounted for [10]. Using the coefficients of [10] for E2/M1 transitions and two-photon induced light shifts (hyperpolarisability) [12], these higher-order effects cause shifts of $0(3.4) \times 10^{-18}$ and $-3.9(1.8) \times 10^{-18}$, respectively.

Tunnelling: Resonant tunnelling in the lattice causes energy bands with finite widths that can be populated non-uniformly, which in turn causes a tunnelling-induced frequency shift. Bandwidths and corresponding shifts can be reduced by a tilt or acceleration of the lattice such that tunnelling is no longer resonant $[13,14]$. As we prepare the atoms almost exclusively in the vibrational ground state in longitudinal direction and operate in a deep lattice, even a small lattice tilt of $\theta=0.12(5)^{\circ}$ present in our set-up is sufficient to reduce the uncertainty due to tunnelling to $2.1 \times 10^{-18}$.

Probe light shift: The highly advanced interrogation laser [5] enables long interrogation times of $640 \mathrm{~ms}$. Therefore, the necessary intensity to drive a $\pi$-pulse and thus the light shift caused by the clock laser are very small. With the coefficient published in [15], we find less than $1 \times 10^{-19}$ of fractional shift, which we use as uncertainty.

Optical path length: Changes of the optical path length between atoms and the clock laser cause a first-order Doppler shift and therefore errors in the frequency measurement. Although continuous drifts due to, e.g., slow temperature changes may not be overly harmful [16], clock-cycle-synchronous vibrations or rf heating of the AOM may. Lattice clocks offer the unique opportunity to stabilize the optical path length from the interrogation laser to the atom position determined by the mirror that forms the standing wave of the 1D lattice. Evaluation of the lock signal [16] reveals an upper limit of path length-induced shifts of $1 \times 10^{-19}$.

Servo error: In practice, all reference cavities used to build narrow bandwidth lasers exhibit a frequency drift due to ageing of the resonator materials and residual temperature variations. Any drift of the clock laser frequency causes a servo error, since frequency corrections are applied discontinuously. We reduce this effect by continuously sweeping the laser frequency with a rate that is updated from the atomic signal. Then, only nonlinearities in the drift cause a servo error. As our new reference cavity shows an exceptionally linear drift, the fractional uncertainty is $1.7 \times 10^{-18}$.

DC Stark shift: Static electric fields in the interrogation region, for instance produced by patch charges on isolating surfaces, can cause substantial frequency shifts [16]. We have evaluated possible offset fields from the analysis of the observed Stark shift induced by intentionally applied electric fields. With an upper limit for the offset field of about $16 \mathrm{~V} / \mathrm{m}$, we expect a DC Stark shift of below $3 \times 10^{-19}$, which we take as uncertainty [2].

During the measurement campaign performed in October 2014 we observed an increased temperature gradient across the apparatus resulting in a larger uncertainty of the Stark shift due to BBR. This lead to a total clock uncertainty of $3 \times 10^{-17}$.

Theory of data extrapolation For the analysis we use the normalized frequency fluctuations $y(t)$ defined as

$$
y(t)=\frac{v(t)-v_{0}}{v_{0}}
$$

with the frequency $v(t)$ and the nominal frequency $v_{0}$. These fractional quantities allow to simply compare fluctuations between oscillators of different average frequencies with frequency ratio $\beta=v_{0}^{(a)} / v_{0}^{(b)}$.

$$
\frac{v^{(a)}(t) / v^{(b)}(t)}{v_{0}^{(a)} / v_{0}^{(b)}}-1 \approx \frac{v^{(a)}(t)-\beta v^{(b)}(t)}{v_{0}^{(a)}}=y^{(a)}(t)-y^{(b)}(t)
$$

To express the uncertainty introduced by the extrapolation, we follow an approach similar to the one used to relate Allan deviations and the oscillator frequency noise spectrum (see e.g. [17] 
and references therein). Averaging the flywheel frequency $y(t)$ over one set of time intervals $\mathbb{T}_{i}$ with total duration $T_{i}$ can be written as

$$
\bar{y}_{\mathbb{T}_{i}}=\int_{-\infty}^{\infty} y(t) g_{\mathbb{T}_{i}}(t) \mathrm{d} t
$$

with the weighting function $g_{\mathbb{T}_{i}}(t)$ :

$$
g_{\mathbb{T}_{i}}(t)=\left\{\begin{array}{ll}
1 / T_{i} & \text { for } t \in \mathbb{T}_{i} \\
0 & \text { elsewhere }
\end{array} .\right.
$$

A similar approach is used for atomic clocks to describe the atoms' response to frequency fluctuations of the interrogation oscillator [18]. Replacing the weighting function in Eq. 3 with the combined one $g(t)=g_{\mathbb{T}_{1}}(t)-g_{\mathbb{T}_{2}}(t)$ yields the difference $\delta y_{\text {ext }}=\bar{y}_{\mathbb{T}_{1}}-\bar{y}_{\mathbb{T}_{2}}$ of the mean flywheel frequencies averaged over the two sets of time intervals $\mathbb{T}_{1}$ and $\mathbb{T}_{2}$.

The uncertainty $u_{\text {ext }}$ due to extrapolating the mean flywheel frequency from $\mathbb{T}_{1}$ to $\mathbb{T}_{2}$ is given by the standard deviation of this difference $\delta y_{\text {ext }}$ :

$$
u_{\mathrm{ext}}^{2}=\left\langle\left(\delta y_{\mathrm{ext}}\right)^{2}\right\rangle
$$

Parseval's theorem relates this variance to the single-sided power spectral density $S(f)$ of the flywheel oscillator's frequency fluctuations as:

$$
u_{\mathrm{ext}}^{2}=\int_{0}^{\infty} S(f)|G(f)|^{2} \mathrm{~d} f,
$$

with $G(f)=\int_{-\infty}^{\infty} g(t) \exp (-2 \pi \mathrm{i} f t) \mathrm{d} t$ being the Fourier transform of the weighting function. Note that the intervals $\mathbb{T}_{1}$ and $\mathbb{T}_{2}$ can deliberately be chosen symmetric about their common center-of-gravity. In this case, a pure linear drift of $y(t)$ will lead to a coincidence of $\bar{y}_{\mathbb{T}_{1}}$ and $\bar{y}_{\mathbb{T}_{2}}$. Thus, $\delta y_{\text {ext }}$ and $u_{\text {ext }}$ will not be affected by the linear drift of the flywheel oscillator.

Model of maser spectrum Based on the observed maser frequency fluctuations after removing a linear frequency drift of $1.1 \times 10^{-16} /$ day, the fractional instability of the maser $\sigma_{\mathrm{H}}(\tau)$ was modelled by three noise contributions that have been added in quadrature: A contribution of $1.18 \times 10^{-13} \cdot \tau^{-1}$ from flicker phase noise, a white frequency noise part of $3.5 \times 10^{-14} \cdot \tau^{-1 / 2}$ and a flicker floor of $3 \times 10^{-16} \cdot \tau^{0}[19,20]$. These values were converted to corresponding spectral power densities of frequency fluctuations [17] that were used in the calculation of $u_{\text {ext }}$ using Eq. 6.

Numerical timescale representation We numerically generate frequency data traces [21] on a $10 \mathrm{~s}$ grid for the maser flywheel and the Sr clock using the time intervals of the measurement campaign. While the clock trace accounts for the statistical noise $\sigma_{\mathrm{Sr}}$, the maser trace reflects the noise and drift characteristics of our maser model (see above).

This data is used to simulate a timescale realization $\mathrm{TS}(\mathrm{Sr})$ according to the steering algorithm. At the beginning of the measurement interval, the time error $\Delta T$ between the timescale and an ideal one is set to zero. To reflect the lack of knowledge about the maser's initial fractional frequency offset we arbitrarily choose it to $5 \times 10^{-16}$ leading to a time error of about $100 \mathrm{ps}$ after two days. To attain a complete picture, one also has to account for the Sr clock's systematics $u_{\mathrm{B}}(\mathrm{Sr})$. This is achieved by setting off the fractional clock frequency from its ideal value by $0.5 \times u_{\mathrm{B}}(\mathrm{Sr})$, which results in a minor time error of about $15 \mathrm{ps}$ (20 ps) over the 12 days ( 25 days).

\section{REFERENCES}

1. H. Katori, M. Takamoto, V. G. Pal'chikov, and V. D. Ovsiannikov, "Ultrastable optical clock with neutral atoms in an engineered light shift trap," Phys. Rev. Lett. 91, 173005-1-4 (2003).

2. S. Falke, N. Lemke, C. Grebing, B. Lipphardt, S. Weyers, V. Gerginov, N. Huntemann, C. Hagemann, A. Al-Masoudi, S. Häfner, S. Vogt, U. Sterr, and C. Lisdat, "A strontium lattice clock with $3 \times 10^{-17}$ inaccuracy and its frequency," New J. Phys. 16, 073023 (2014).

3. T. Kessler, C. Hagemann, C. Grebing, T. Legero, U. Sterr, F. Riehle, M. J. Martin, L. Chen, and J. Ye, "A sub-40-mHzlinewidth laser based on a silicon single-crystal optical cavity," Nature Photonics 6, 687-692 (2012).

4. C. Hagemann, C. Grebing, T. Kessler, S. Falke, N. Lemke, C. Lisdat, H. Schnatz, F. Riehle, and U. Sterr, "Providing $10^{-16}$ short-term stability of a $1.5 \mu \mathrm{m}$ laser to optical clocks," IEEE Trans. Instrum. Meas. 62, 1556-1562 (2013).

5. S. Häfner, S. Falke, C. Grebing, S. Vogt, T. Legero, M. Merimaa, C. Lisdat, and U. Sterr, " $8 \times 10^{-17}$ fractional laser frequency instability with a long room-temperature cavity," Opt. Lett. 40, 2112-2115 (2015).

6. T. Middelmann, C. Lisdat, S. Falke, J. S. R. Vellore Winfred, F. Riehle, and U. Sterr, "Tackling the blackbody shift in a strontium optical lattice clock," IEEE Trans. Instrum. Meas. 60, 2550-2557 (2011).

7. M. S. Safronova, S. G. Porsev, U. I. Safronova, M. G. Kozlov, and C. W. Clark, "Blackbody radiation shift in the Sr optical atomic clock," Phys. Rev. A 87, 012509 (2013).

8. I. Ushijima, M. Takamoto, M. Das, T. Ohkubo, and H. Katori, "Cryogenic optical lattice clocks," Nature Photonics 9, 185189 (2015).

9. T. L. Nicholson, S. L. Campbell, R. B. Hutson, G. E. Marti, B. J. Bloom, R. L. McNally, W. Zhang, M. D. Barrett, M. S. Safronova, G. F. Strouse, W. L. Tew, and J. Ye, "Systematic evaluation of an atomic clock at $2 \times 10^{-18}$ total uncertainty," Nature Com. 6, 6896 (2015).

10. P. G. Westergaard, J. Lodewyck, L. Lorini, A. Lecallier, E. A. Burt, M. Zawada, J. Millo, and P. Lemonde, "Latticeinduced frequency shifts in Sr optical lattice clocks at the 10-17 level," Phys. Rev. Lett. 106, 210801 (2011).

11. K. Gibble, "Scattering of cold atom coherences by hot atoms: Frequency shifts from background gas collisions," Phys. Rev. Lett. 110, 180802 (2013).

12. R. Le Targat, L. Lorini, Y. Le Coq, M. Zawada, J. Guéna, M. Abgrall, M. Gurov, P. Rosenbusch, D. G. Rovera, B. Nagórny, R. Gartman, P. G. Westergaard, M. E. Tobar, M. Lours, G. Santarelli, A. Clairon, S. Bize, P. Laurent, P. Lemonde, and J. Lodewyck, "Experimental realization of an optical second with strontium lattice clocks," Nature Com. 4, 2109 (2013).

13. P. Lemonde and P. Wolf, "Optical lattice clock with atoms confined in a shallow trap," Phys. Rev. A 72, 033409 (2005).

14. C. Sias, H. Lignier, Y. P. Singh, A. Zenesini, D. Ciampini, O. Morsch, and E. Arimondo, "Observation of photonassisted tunneling in optical lattices," Phys. Rev. Lett. 100, 040404 (2008).

15. X. Baillard, M. Fouché, R. Le Targat, P. G. Westergaard, A. Lecallier, Y. Le Coq, G. D. Rovera, S. Bize, and P. Lemonde, "Accuracy evaluation of an optical lattice clock with bosonic atoms," Opt. Lett. 32, 1812-1814 (2007).

16. S. Falke, M. Misera, U. Sterr, and C. Lisdat, “Delivering 
pulsed and phase stable light to atoms of an optical clock," Appl. Phys. B 107, 301-311 (2012).

17. S. T. Dawkins, J. J. McFerran, and A. N. Luiten, "Considerations on the measurement of the stability of oscillators with frequency counters," IEEE Trans. Ultrason. Ferroelectr. Freq. Control 54, 918-925 (2007).

18. G. J. Dick, "Local oscillator induced instabilities in trapped ion frequency standards," in "Proceedings of $19^{\text {th }}$ Annu. Precise Time and Time Interval Meeting, Redendo Beach, 1987," (U.S. Naval Observatory, Washington, DC, 1988), pp. 133-147.

19. T. E. Parker, "Hydrogen maser ensemble performance and characterization of frequency standards," in "Proceedings of the 1999 Joint Meeting of the European Frequency and Time Forum and The IEEE International Frequency Control Symposium," (1999), pp. 173-176.

20. V. Vorontsov, A. Belyaev, and N. Demidov, "Design of the active hydrogen maser new model (VCH-1003M) using microwave cavity frequency switching technique for cavity auto tuning," in "Materials of the VI symposium "metrology of time and space" 2012," (2012).

21. "Stable32: Frequency stability analysis," Software version 1.20, Hamilton Technical Services, Beaufort, SC 29907, USA (1998). 